\title{
Optimal decay rates for the acoustic wave motions with boundary memory damping
}

\author{
Khalida Benomar and Abbes Benaissa
}

\begin{abstract}
A linear wave equation with acoustic boundary conditions (ABC) on a portion of the boundary and Dirichlet conditions on the rest of the boundary is considered. The $(\mathrm{ABC})$ contain a memory damping with respect to the normal displacement of the boundary point. In this paper, we establish polynomial energy decay rates for the wave equation by using resolvent estimates.
\end{abstract}

Mathematics Subject Classification (2010): 93D15, 35B40, 47D03, 74D05.

Keywords: Wave equation, acoustic boundary conditions, boundary memory.

\section{Introduction}

In this paper we investigate the existence and decay properties of solutions for the initial boundary value problem of the wave equation of the type

$$
\begin{cases}y_{t t}(x, t)-y_{x x}(x, t)=0 & \text { in }(0, L) \times(0,+\infty), \\ y(0, t)=0 & \text { in }(0,+\infty), \\ y_{x}(L, t)=z_{t}(t) & \text { in }(0,+\infty), \\ y_{t}(L, t)+m z(t)+\gamma \partial_{t}^{\alpha, \eta} z(t)=0 & \text { in }(0,+\infty), \\ y(x, 0)=y_{0}(x), \quad y_{t}(x, 0)=y_{1}(x) & \text { in }(0, L),\end{cases}
$$

where $(x, t) \in(0, L) \times(0,+\infty), m>0, \gamma>0, \eta \geq 0$ and the initial data are taken in suitable spaces. The notation $\partial_{t}^{\alpha, \eta}$ stands for the generalized Caputo's fractional derivative of order $\alpha, 0<\alpha<1$, with respect to the time variable (see Choi and MacCamy [9]). It is defined as follows

$$
\partial_{t}^{\alpha, \eta} w(t)=\frac{1}{\Gamma(1-\alpha)} \int_{0}^{t}(t-s)^{-\alpha} e^{-\eta(t-s)} \frac{d w}{d s}(s) d s, \quad \eta \geq 0 .
$$

The problem $(P)$ describes sound wave propagation in a domain which is full of some kind of medium and with a portion of boundary made of light-weight viscoelastic material. 
Acoustic model was proposed by Morse and Ingard [15], and improved in a rigorous mathematical way by Beale and Rosencrans [5]. Under the assumption that each local-reacting boundary point acts as a spring, the author analyzed the model in both bounded and exterior domains in [3], [4]. Uniform energy decay rates were studied in [7], [16] for acoustic wave systems with both internal and boundary memory damping terms. To our knowledge, there has been few work about the decay rates of acoustic wave energies when only one memory damping acting on the acoustic boundary.

Recently, In [11], the authors considered the following initial boundary value problem with memory type acoustic boundary conditions,

$$
\begin{cases}y_{t t}(x, t)-\Delta y(x, t)=0 & \text { in } \Omega \times(0,+\infty), \\ y(x, t)=0 & \text { in } \Gamma_{0} \times(0,+\infty), \\ \frac{\partial y}{\partial \nu}(x, t)=z_{t}(x, t) & \text { in } \Gamma_{1} \times(0,+\infty), \\ y_{t}(x, t)+m z(x, t)+\gamma \partial_{t}^{\alpha, 0} z(x, t)=0 & \text { in } \Gamma_{1} \times(0,+\infty), \\ y(x, 0)=y_{0}(x), \quad y_{t}(x, 0)=y_{1}(x) & \text { in }(0, L) .\end{cases}
$$

They proved well-posedness and strong stability of the system $(P)$ without giving an energy decay rate. Very Recently, in [12] the authors proved that the energy is polynomially stable but without obtaining the precise exponent.

The aim of the present paper is to obtain more precise rates of decay. This can be achieved via some theorems about operator semigroups. We provide a standard method of going from resolvent estimates for a suitable PDE to rates of decay of classical (strong) solutions.

We should mention here that the approach in [11] and [12], which is based on Laplace transform is different from ours. By redescribing the fractional derivative term by means of a suitable diffusion equation as in [14], the original model is transformed into an augmented system which can be more easily tackled by the energy method.

\section{Augmented model}

This section is concerned with the reformulation of the model $(P)$ into an augmented system. For that, we need the following claims.

Theorem 2.1 (see [14]). Let $\mu$ be the function:

$$
\mu(\xi)=|\xi|^{(2 \alpha-1) / 2}, \quad-\infty<\xi<+\infty, 0<\alpha<1 .
$$

Then the relationship between the 'input' $U$ and the 'output' $O$ of the system

$$
\begin{gathered}
\partial_{t} \phi(\xi, t)+\left(\xi^{2}+\eta\right) \phi(\xi, t)-U(t) \mu(\xi)=0, \quad-\infty<\xi<+\infty, \eta \geq 0, t>0, \\
\phi(\xi, 0)=0, \\
O(t)=(\pi)^{-1} \sin (\alpha \pi) \int_{-\infty}^{+\infty} \mu(\xi) \phi(\xi, t) d \xi
\end{gathered}
$$

is given by

$$
O=I^{1-\alpha, \eta} U=D^{\alpha, \eta} U
$$


where

$$
\left[I^{\alpha, \eta} f\right](t)=\frac{1}{\Gamma(\alpha)} \int_{0}^{t}(t-\tau)^{\alpha-1} e^{-\eta(t-\tau)} f(\tau) d \tau .
$$

Lemma 2.2 (see [1]). If $\left.\left.\lambda \in D_{\eta}=\mathbb{C} \backslash\right]-\infty,-\eta\right]$ then

$$
\int_{-\infty}^{+\infty} \frac{\mu^{2}(\xi)}{\lambda+\eta+\xi^{2}} d \xi=\frac{\pi}{\sin \alpha \pi}(\lambda+\eta)^{\alpha-1}
$$

We are now in a position to reformulate system $(P)$. Indeed, by using Theorem 2.1, system $(P)$ may be recast into the augmented model:

$$
\begin{cases}y_{t t}(x, t)-y_{x x}(x, t)=0 & \text { in }(0, L) \times(0,+\infty), \\ \partial_{t} \phi(\xi, t)+\left(\xi^{2}+\eta\right) \phi(\xi, t)-z_{t}(t) \mu(\xi)=0 & \text { in }(-\infty,+\infty) \times(0,+\infty), \\ y(0, t)=0 & \text { in }(0,+\infty), \\ y_{x}(L, t)=z_{t}(t) & \text { in }(0,+\infty), \\ y_{t}(L, t)+m z(t)+\zeta \int_{-\infty}^{+\infty} \mu(\xi) \phi(\xi, t) d \xi=0 & \text { in }(0,+\infty), \\ y(x, 0)=y_{0}(x), \quad y_{t}(x, 0)=y_{1}(x) & \text { in }(0, L), \\ \phi(\xi, 0)=0 & \text { in }(-\infty,+\infty) .\end{cases}
$$

We define the energy associated to the solution of the problem $\left(P^{\prime}\right)$ by the following formula:

$$
E(t)=\frac{1}{2}\left\|y_{t}\right\|_{2}^{2}+\frac{1}{2}\left\|y_{x}\right\|_{2}^{2}+\frac{m}{2}|z(t)|^{2}+\frac{\zeta}{2} \int_{-\infty}^{+\infty}|\phi(\xi, t)|^{2} d \xi .
$$

Lemma 2.3. Let $(y, \phi)$ be a solution of the problem $\left(P^{\prime}\right)$. Then, the energy functional defined by (2.6) satisfies

$$
E^{\prime}(t)=-\zeta \int_{-\infty}^{+\infty}\left(\xi^{2}+\eta\right)|\phi(\xi, t)|^{2} d \xi \leq 0
$$

Proof. Multiplying the first equation in $\left(P^{\prime}\right)$ by $\bar{y}_{t}$, integrating over $(0, L)$ and using integration by parts, we get

$$
\frac{1}{2} \frac{d}{d t}\left\|y_{t}\right\|_{2}^{2}-\Re \int_{0}^{L} y_{x x} \bar{y}_{t} d x=0 .
$$

Then

$$
\frac{d}{d t}\left(\frac{1}{2}\left\|y_{t}\right\|_{2}^{2}+\frac{1}{2}\left\|y_{x}\right\|_{2}^{2}\right)+\Re z_{t}(t)\left(m \bar{z}(t)+\zeta \int_{-\infty}^{+\infty} \mu(\xi) \bar{\phi}(\xi, t) d \xi\right)=0 .
$$

Multiplying the second equation in $\left(P^{\prime}\right)$ by $\zeta \bar{\phi}_{t}$ and integrating over $(-\infty,+\infty)$, to obtain:

$$
\frac{\zeta}{2} \frac{d}{d t}\|\phi\|_{2}^{2}+\zeta \int_{-\infty}^{+\infty}\left(\xi^{2}+\eta\right)|\phi(\xi, t)|^{2} d \xi-\zeta \Re z_{t}(t) \int_{-\infty}^{+\infty} \mu(\xi) \bar{\phi}(\xi, t) d \xi=0
$$

From (2.6), (2.8) and (2.9) we get

$$
E^{\prime}(t)=-\zeta \int_{-\infty}^{+\infty}\left(\xi^{2}+\eta\right)|\phi(\xi, t)|^{2} d \xi
$$

This completes the proof of the lemma. 


\section{Well-posedness}

The energy space associated to system $(P)$ is

$\mathcal{H}=H_{L}^{1}(0, L) \times L^{2}(0, L) \times L^{2}(-\infty,+\infty) \times \mathbb{C}, \quad H_{L}^{1}(0, L)=\left\{y \in H^{1}(0, L), y(0)=0\right\}$ equipped with the inner product

$$
<U, \tilde{U}>_{\mathcal{H}}=\int_{\Omega}\left(v \overline{\tilde{v}}+y_{x} \overline{\tilde{y}}_{x}\right) d x+m z \overline{\tilde{z}}+\zeta \int_{-\infty}^{+\infty} \phi \overline{\tilde{\phi}} d \xi,
$$

where $U=(y, v, \phi, z)^{T}, \tilde{U}=(\tilde{y}, \tilde{v}, \tilde{\phi}, \tilde{z})^{T} \in \mathcal{H}$.

Let $U=\left(y, y_{t}, \phi, z\right)^{T}$ and rewrite $\left(P^{\prime}\right)$ as

$$
\left\{\begin{array}{l}
U^{\prime}=\mathcal{A} U \\
U(0)=\left(y_{0}, y_{1}, \phi_{0}, z_{0}\right),
\end{array}\right.
$$

where the operator $\mathcal{A}$ is defined by

with domain

$$
\mathcal{A}\left(\begin{array}{c}
y \\
v \\
\phi \\
z
\end{array}\right)=\left(\begin{array}{c}
v \\
y_{x x} \\
-\left(\xi^{2}+\eta\right) \phi+y_{x}(L) \mu(\xi) \\
y_{x}(L)
\end{array}\right)
$$

$$
D(\mathcal{A})=\left\{\begin{array}{l}
(y, v, \phi, z)^{T} \text { in } \mathcal{H}: y \in H^{2}(0, L) \cap H_{L}^{1}(0, L), \\
v \in H_{L}^{1}(0, L), z \in \mathbb{C} \\
-\left(\xi^{2}+\eta\right) \phi+y_{x}(L) \mu(\xi) \in L^{2}(-\infty,+\infty), \\
v(L)+m z+\zeta \int_{-\infty}^{\infty} \mu(\xi) \phi(\xi) d \xi=0 \\
|\xi| \phi \in L^{2}(-\infty,+\infty)
\end{array}\right\}
$$

Now, we will give well-posedness results for problem $(P)$ using semigroup theory. We show that the operator $\mathcal{A}$ generates a $C_{0^{-}}$semigroup in $\mathcal{H}$. We prove that $\mathcal{A}$ is a maximal dissipative operator (see [8]). For this purpose we need the following two lemmas.

Lemma 3.1. The operator $\mathcal{A}$ is dissipative and satisfies, for any $U \in D(\mathcal{A})$,

$$
\Re\langle\mathcal{A} U, U\rangle_{\mathcal{H}}=-\zeta \int_{-\infty}^{+\infty}\left(\xi^{2}+\eta\right)|\phi(\xi)|^{2} d \xi .
$$

Proof. For any $U=(y, v, \phi, z)^{T} \in D(A)$, Using (3.1) and the fact that

$$
\left\|\left(y, y_{t}, \phi, z\right)\right\|_{\mathcal{H}}^{2}=\|U\|_{\mathcal{H}}^{2},
$$

estimate (3.4) easily follows.

Lemma 3.2. The operator $\lambda I-\mathcal{A}$ is surjective for all $\lambda>0$.

Proof. We need to show that for all $F=\left(f_{1}, f_{2}, f_{3}, f_{4}\right)^{T} \in \mathcal{H}$, there exists

$$
U=(y, u, \phi, v)^{T} \in D(\mathcal{A})
$$

such that

$$
\lambda U-\mathcal{A} U=F,
$$


that is

$$
\left\{\begin{array}{l}
\lambda y-v=f_{1} \\
\lambda v-y_{x x}=f_{2} \\
\lambda \phi+\left(\xi^{2}+\eta\right) \phi-y_{x}(L) \mu(\xi)=f_{3} \\
\lambda z-y_{x}(L)=f_{4}
\end{array}\right.
$$

Suppose that we have found $y$. Therefore, the first equation in (3.7) gives

$$
v=\lambda y-f_{1} .
$$

It is clear that $u \in H_{L}^{1}(0, L)$. Furthermore, by (3.7) we can find $\phi$ as

$$
\phi=\frac{f_{3}(\xi)+\mu(\xi) y_{x}(L)}{\xi^{2}+\eta+\lambda} .
$$

By using (3.7) and (3.8) the function $y$ satisfying the following system

$$
\lambda^{2} y-y_{x x}=f_{2}+\lambda f_{1} .
$$

Solving system (3.10) is equivalent to finding $y \in H^{2} \cap H_{L}^{1}(0, L)$ such that

$$
\int_{0}^{L}\left(\lambda^{2} y \bar{w}-y_{x x} \bar{w}\right) d x=\int_{0}^{L}\left(f_{2}+\lambda f_{1}\right) \bar{w} d x
$$

for all $w \in H_{L}^{1}(0, L)$. By using (3.11) and (3.9) the function $y$ satisfying the following system

$$
\left\{\begin{aligned}
\int_{0}^{L}\left(\lambda^{2} y \bar{w}+y_{x} \bar{w}_{x}\right) d x+\frac{\lambda^{2}}{m+\gamma \lambda(\lambda+\eta)^{\alpha-1}} y(L) \bar{w}(L) \\
=\int_{0}^{L}\left(f_{2}+\lambda f_{1}\right) \bar{w} d x+\frac{1}{m+\gamma \lambda(\lambda+\eta)^{\alpha-1}}\left(\lambda f_{1}(L)\right. \\
-\zeta \lambda \int_{-\infty}^{+\infty} \frac{\mu(\xi)}{\xi^{2}+\eta+\lambda} f_{3}(\xi) d \xi-m f_{4} \bar{w}(L) .
\end{aligned}\right.
$$

Consequently, problem (3.12) is equivalent to the problem

$$
a(y, w)=L(w)
$$

where the sesquilinear form $a: H_{L}^{1}(0, L) \times H_{L}^{1}(0, L) \rightarrow \mathbb{C}$ and the antilinear form $L: H_{L}^{1}(0, L) \rightarrow \mathbb{C}$ are defined by

$$
a(y, w)=\int_{0}^{L}\left(\lambda^{2} y \bar{w}+y_{x} \bar{w}_{x}\right) d x+\frac{\lambda^{2}}{m+\gamma \lambda(\lambda+\eta)^{\alpha-1}} y(L) \bar{w}(L)
$$

and

$$
\begin{aligned}
L(w)=\int_{0}^{L}\left(f_{2}+\lambda f_{1}\right) \bar{w} d x & +\frac{1}{m+\gamma \lambda(\lambda+\eta)^{\alpha-1}}\left(\lambda f_{1}(L)\right. \\
& -\zeta \lambda \int_{-\infty}^{+\infty} \frac{\mu(\xi)}{\xi^{2}+\eta+\lambda} f_{3}(\xi) d \xi-m f_{4} \bar{w}(L) .
\end{aligned}
$$

It is easy to verify that $a$ is continuous and coercive, and $L$ is continuous. So applying the Lax-Milgram theorem, we deduce that for all $w \in H_{L}^{1}(0, L)$ problem (3.13) admits a unique solution $y \in H_{L}^{1}(0, L)$. Applying the classical elliptic regularity, it follows from (3.12) that $y \in H^{2}(0, L)$. Therefore, the operator $\lambda I-\mathcal{A}$ is surjective for any $\lambda>0$. 
Consequently, using Hille-Yosida Theorem, we have the following well-posedness result:

\section{Theorem 3.3 (Existence and uniqueness).}

(1) If $U_{0} \in D(\mathcal{A})$, then system (3.1) has a unique strong solution

$$
U \in C^{0}\left(\mathbb{R}_{+}, D(\mathcal{A})\right) \cap C^{1}\left(\mathbb{R}_{+}, \mathcal{H}\right) .
$$

(1) If $U_{0} \in \mathcal{H}$, then system (3.1) has a unique weak solution

$$
U \in C^{0}\left(\mathbb{R}_{+}, \mathcal{H}\right) .
$$

\section{Lack of exponential stability}

In order to state and prove our stability results, we need the following well known theorems.

Theorem 4.1 ([17]-[10]). Let $S(t)=e^{\mathcal{A} t}$ be a $C_{0}$-semigroup of contractions on Hilbert space $\mathcal{H}$. Then $S(t)$ is exponentially stable if and only if

$$
\rho(\mathcal{A}) \supseteq\{i \beta: \beta \in \mathbb{R}\} \equiv i \mathbb{R}
$$

and

$$
\overline{\lim }_{|\beta| \rightarrow \infty}\left\|(i \beta I-\mathcal{A})^{-1}\right\|_{\mathcal{L}(\mathcal{H})}<\infty .
$$

Theorem $4.2([6])$. Let $S(t)=e^{\mathcal{A} t}$ be a $C_{0}$-semigroup on a Hilbert space $\mathcal{H}$. If

$$
i \mathbb{R} \subset \rho(\mathcal{A}) \text { and } \sup _{|\beta| \geq 1} \frac{1}{\beta^{\delta}}\left\|(i \beta I-\mathcal{A})^{-1}\right\|_{\mathcal{L}(\mathcal{H})}<M
$$

for some $\delta>0$, then there exist c such that

$$
\left\|e^{\mathcal{A} t} U_{0}\right\|^{2} \leq \frac{c}{t^{\frac{2}{\delta}}}\left\|U_{0}\right\|_{D(\mathcal{A})}^{2} .
$$

Theorem 4.3 ([2]-[13]). Let $\mathcal{A}$ be the generator of a uniformly bounded $C_{0}$ - semigroup $\{S(t)\}_{t \geq 0}$ on a Hilbert space $\mathcal{H}$. If:

(i) $\mathcal{A}$ does not have eigenvalues on $i \mathbb{R}$.

(ii) The intersection of the spectrum $\sigma(\mathcal{A})$ with $i \mathbb{R}$ is at most a countable set, then the semigroup $\{S(t)\}_{t \geq 0}$ is asymptotically stable, i.e, $\|S(t) z\|_{\mathcal{H}} \rightarrow 0$ as $t \rightarrow \infty$ for any $z \in \mathcal{H}$.

Our main first result is

Theorem 4.4. The semigroup generated by the operator $\mathcal{A}$ is not exponentially stable.

Proof. We will examine two cases.

- Case 1. $\eta=0$ : We shall show that $i \lambda=0$ is not in the resolvent set of the operator $\mathcal{A}$. Indeed, noting that $(0,0,0, \cos L)^{T} \in \mathcal{H}$, and denoting by $(y, v, \phi, z)^{T}$ the image of $(0,0,0, \cos L)^{T}$ by $\mathcal{A}^{-1}$, we see that $\phi(\xi)=|\xi|^{\frac{2 \alpha-5}{2}} \cos L$. But, then $\phi \notin L^{2}(-\infty,+\infty)$, since $\alpha \in] 0,1\left[\right.$. Hence $(y, v, \phi, z)^{T} \notin D(\mathcal{A})$.

- Case 2. $\eta \neq 0$ : We aim to show that an infinite number of eigenvalues of $\mathcal{A}$ approach the imaginary axis which prevents the wave system $(P)$ from being exponentially 
stable. Indeed we first compute the characteristic equation that gives the eigenvalues of $\mathcal{A}$. Let $\lambda$ be an eigenvalue of $\mathcal{A}$ with associated eigenvector $U=(y, v, \phi, z)^{T}$. Then $\mathcal{A} U=\lambda U$ is equivalent to

$$
\left\{\begin{array}{l}
\lambda y-v=0 \\
\lambda v-y_{x x}=0 \\
\lambda \phi+\left(\xi^{2}+\eta\right) \phi-y_{x}(L) \mu(\xi)=0 \\
\lambda z-y_{x}(L)=0 \\
v(L)+m z+\zeta \int_{-\infty}^{+\infty} \mu(\xi) \phi(\xi) d \xi=0
\end{array}\right.
$$

From $(4.1)_{1}-(4.1)_{2}$ for such $\lambda$, we find

$$
\lambda^{2} y-y_{x x}=0 .
$$

Since $v=\lambda y(L)$, using $(4.1)_{3}$ and $(4.1)_{4}$, we get

$$
\left\{\begin{array}{l}
y(0)=0, \\
\lambda^{2} y(L)+\left(m+\gamma \lambda(\lambda+\eta)^{\alpha-1}\right) y_{x}(L)=0 .
\end{array}\right.
$$

The solution $y$ is given by

$$
y(x)=\sum_{i=1}^{2} c_{i} e^{t_{i} x}
$$

where

$$
t_{1}(\lambda)=\lambda, \quad t_{2}(\lambda)=-\lambda .
$$

Thus the boundary conditions may be written as the following system:

$$
M(\lambda) C(\lambda)=\left(\begin{array}{cc}
1 & 1 \\
h\left(t_{1}\right) e^{t_{1} L} & h\left(t_{2}\right) e^{t_{2} L}
\end{array}\right)\left(\begin{array}{l}
c_{1} \\
c_{2}
\end{array}\right)=\left(\begin{array}{l}
0 \\
0
\end{array}\right)
$$

where we have set

$$
h(r)=\left(m+\gamma \lambda(\lambda+\eta)^{\alpha-1}\right) r+\lambda^{2} .
$$

Hence a non-trivial solution $y$ exists if and only if the determinant of $M(\lambda)$ vanishes. Set $f(\lambda)=\operatorname{det} M(\lambda)$, thus the characteristic equation is $f(\lambda)=0$.

Our purpose is to prove, thanks to Rouché's Theorem, that there is a subsequence of eigenvalues for which their real part tends to 0 .

In the sequel, since $\mathcal{A}$ is dissipative, we study the asymptotic behavior of the large eigenvalues $\lambda$ of $\mathcal{A}$ in the strip $-\alpha_{0} \leq \mathcal{R}(\lambda) \leq 0$, for some $\alpha_{0}>0$ large enough and for such $\lambda$, we remark that $e^{t_{i}}, i=1,2$ remains bounded.

Lemma 4.5. There exists $N \in \mathbb{N}$ such that

$$
\left\{\lambda_{k}\right\}_{k \in \mathbb{Z}^{*},|k| \geq N} \subset \sigma(\mathcal{A})
$$

where

$$
\begin{gathered}
\lambda_{k}=i \frac{k \pi}{L}+\frac{\tilde{\alpha}}{k^{1-\alpha}}+\frac{\beta}{k^{1-\alpha}}+o\left(\frac{1}{k^{1-\alpha}}\right), k \geq N, \tilde{\alpha} \in i \mathbb{R}, \beta \in \mathbb{R}, \beta<0, \\
\lambda_{k}=\overline{\lambda_{-k}} \text { if } k \leq-N .
\end{gathered}
$$

Moreover for all $|k| \geq N$, the eigenvalues $\lambda_{k}$ are simple. 


\section{Proof. Step 1.}

$$
\begin{aligned}
f(\lambda) & =e^{t_{2}} h\left(t_{2}\right)-e^{t_{1}} h\left(t_{1}\right) \\
& =-e^{-\lambda L}\left(\left(m+\gamma \lambda(\lambda+\eta)^{\alpha-1}\right)+\lambda\right)\left(e^{2 \lambda L}-\frac{\lambda-\left(m+\gamma \lambda(\lambda+\eta)^{\alpha-1}\right)}{\lambda+\left(m+\gamma \lambda(\lambda+\eta)^{\alpha-1}\right)}\right) \\
& =-e^{-\lambda L}\left(\left(m+\gamma \lambda(\lambda+\eta)^{\alpha-1}\right)+\lambda\right)\left(e^{2 \lambda L}-1+2 \frac{m+\gamma \lambda(\lambda+\eta)^{\alpha-1}}{m+\lambda+\gamma \lambda(\lambda+\eta)^{\alpha-1}}\right) .
\end{aligned}
$$

We set

$$
\begin{aligned}
\tilde{f}(\lambda) & =e^{2 \lambda L}-1+2 \frac{m+\gamma \lambda(\lambda+\eta)^{\alpha-1}}{m+\lambda+\gamma \lambda(\lambda+\eta)^{\alpha-1}} \\
& =f_{0}(\lambda)+\frac{f_{1}(\lambda)}{\lambda^{1-\alpha}}+o\left(\frac{1}{\lambda^{1-\alpha}}\right)
\end{aligned}
$$

where

$$
\begin{gathered}
f_{0}(\lambda)=e^{2 \lambda L}-1, \\
f_{1}(\lambda)=2 \gamma .
\end{gathered}
$$

Note that $f_{0}$ and $f_{1}$ remain bounded in the strip $-\alpha_{0} \leq \mathcal{R}(\lambda) \leq 0$.

Step 2. We look at the roots of $f_{0}$. From (4.9), $f_{0}$ has one familie of roots that we denote $\lambda_{k}^{0}$.

$$
f_{0}(\lambda)=0 \Leftrightarrow e^{2 \lambda L}=1
$$

Hence

$$
2 \lambda L=i 2 k \pi, \quad k \in \mathbb{Z}
$$

i.e.,

$$
\lambda_{k}^{0}=\frac{i k \pi}{L}, \quad k \in \mathbb{Z} .
$$

Now with the help of Rouché's Theorem, we will show that the roots of $\tilde{f}$ are close to those of $f_{0}$. Changing in (4.8) the unknown $\lambda$ by $u=2 \lambda L$ then (4.8) becomes

$$
\tilde{f}(u)=\left(e^{u}-1\right)+O\left(\frac{1}{u}\right)=f_{0}(u)+O\left(\frac{1}{u}\right) .
$$

The roots of $f_{0}$ are $u_{k}=\frac{i k}{L} \pi, k \in \mathbb{Z}$, and setting $u=u_{k}+r e^{i t}, t \in[0,2 \pi]$, we can easily check that there exists a constant $C>0$ independent of $k$ such that $\left|e^{u}-1\right| \geq C r$ for $r$ small enough. This allows to apply Rouché's Theorem. Consequently, there exists a subsequence of roots of $\tilde{f}$ which tends to the roots $u_{k}$ of $f_{0}$. Equivalently, it means that there exists $N \in \mathbb{N}$ and a subsequence $\left\{\lambda_{k}\right\}_{|k| \geq N}$ of roots of $f(\lambda)$, such that $\lambda_{k}=\lambda_{k}^{0}+o(1)$ which tends to the roots $\frac{i k}{L} \pi$ of $f_{0}$. Finally for $|k| \geq N, \lambda_{k}$ is simple since $\lambda_{k}^{0}$ is.

Step 3. From Step 2, we can write

$$
\lambda_{k}=i \frac{1}{L} k \pi+\varepsilon_{k}
$$

Using (4.11), we get

$$
e^{2 \lambda_{k} L}=1+2 L \varepsilon_{k}+2 L^{2} \varepsilon_{k}^{2}+o\left(\varepsilon_{k}^{2}\right) .
$$


Substituting (4.12) into (4.8), using that $\tilde{f}\left(\lambda_{k}\right)=0$, we get:

$$
\begin{aligned}
\tilde{f}\left(\lambda_{k}\right) & =2 L \varepsilon_{k}+\frac{2 \gamma}{\left(\frac{k \pi i}{L}+\varepsilon_{k}\right)^{(1-\alpha)}}+o\left(\varepsilon_{k}\right)+o(1 / k) \\
& =2 L \varepsilon_{k}+\frac{2 \gamma}{\left(\frac{k \pi}{L} i\right)^{(1-\alpha)}}+o\left(\frac{1}{k}\right)=0
\end{aligned}
$$

and hence

$$
\varepsilon_{k}=-\frac{\gamma}{L^{\alpha}} \frac{1}{(k \pi)^{(1-\alpha)}}\left(\cos (1-\alpha) \frac{\pi}{2}-i \sin (1-\alpha) \frac{\pi}{2}\right)+o\left(\frac{1}{k^{1-\alpha}}\right) \text { for } k \succeq 0 .
$$

From (4.13) we have in that case $|k|^{1-\alpha} \mathcal{R} \lambda_{k} \sim \beta$, with

$$
\beta=-\frac{\gamma}{L^{\alpha} \pi^{1-\alpha}} \cos (1-\alpha) \frac{\pi}{2}
$$

The operator $\mathcal{A}$ has a non exponential decaying branch of eigenvalues. Thus the proof is complete.

\section{Polynomial stability and optimality (for $\eta \neq 0$ )}

In the previous section,we have shown that the transmission wave system is not exponentially stable. In this section, we prove that it is polynomially stable with an optimal rate of decay when $\eta>0$. To achieve this, we use a recent result by Borichev and Tomilov [6]. Accordingly, if we consider a bounded $C_{0}$-semigroup $S(t)=e^{\mathcal{A} t}$ on a Hilbert space. If

$$
i \mathbb{R} \subset \rho(\mathcal{A}) \text { and } \varlimsup_{|\beta| \rightarrow \infty} \frac{1}{\beta^{\delta}}\left\|(i \beta I-\mathcal{A})^{-1}\right\|_{\mathcal{L}(\mathcal{H})}<\infty
$$

for some $\delta>0$, then there exist $c$ such that

$$
\left\|e^{\mathcal{A} t} U_{0}\right\|^{2} \leq \frac{c}{t^{\frac{2}{\delta}}}\left\|U_{0}\right\|_{D(\mathcal{A})}^{2}
$$

Our main result is as follows.

Theorem 5.1. The semigroup $S_{\mathcal{A}}(t)_{t \geq 0}$ is polynomially stable and

$$
E(t)=\left\|S_{\mathcal{A}}(t) U_{0}\right\|_{\mathcal{H}}^{2} \leq \frac{1}{t^{2 /(1-\alpha)}}\left\|U_{0}\right\|_{D(\mathcal{A})}^{2} .
$$

Moreover, the rate of energy decay $t^{-2 /(1-\alpha)}$ is optimal for any initial data in $D(\mathcal{A})$. Proof. We will need to study the resolvent equation $(i \lambda-\mathcal{A}) U=F$, for $\lambda \in \mathbb{R}$, namely

$$
\left\{\begin{array}{l}
i \lambda y-v=f_{1} \\
i \lambda v-y_{x x}=f_{2} \\
i \lambda \phi+\left(\xi^{2}+\eta\right) \phi-y_{x}(L) \mu(\xi)=f_{3}, \\
i \lambda z-y_{x}(L)=f_{4}
\end{array}\right.
$$

with the boundary condition

$$
v(L)+m z+\zeta \int_{-\infty}^{+\infty} \mu(\xi) \phi(\xi) d \xi=0
$$


We divide the proof into three steps, as follows:

Step 1. Inserting $(5.1)_{1}$ into $(5.1)_{2}$, we get

$$
\lambda^{2} y+y_{x x}=-\left(f_{2}+i \lambda f_{1}\right)
$$

As $y(0)=0$, then

$$
y(x)=c_{1} \sin \lambda x-\frac{1}{\lambda} \int_{0}^{x}\left(f_{2}(\sigma)+i \lambda f_{1}(\sigma)\right) \sin \lambda(x-\sigma) d \sigma,
$$

and hence

$$
y_{x}(x)=c_{1} \lambda \cos \lambda x-\int_{0}^{x}\left(f_{2}(\sigma)+i \lambda f_{1}(\sigma)\right) \cos \lambda(x-\sigma) d \sigma .
$$

Step 2. With the third equation of (5.1), we get

$$
\phi(\xi)=\frac{y_{x}(L) \mu(\xi)+f_{3}(\xi)}{i \lambda+\xi^{2}+\eta} .
$$

Inserting (5.5) in the boundary condition (5.2), we easy to check that

$$
-\lambda^{2} y(L)+\left(m+\gamma i \lambda(i \lambda+\eta)^{\alpha-1}\right) y_{x}(L)=i \lambda f_{1}(L)-m f_{4}-\zeta i \lambda \int_{-\infty}^{+\infty} \frac{\mu(\xi) f_{3}(\xi)}{i \lambda+\xi^{2}+\eta} d \xi
$$

Using (5.3) and (5.4), we can rewrite (5.6) as an equation in the unknown $c_{1}$,

$$
\begin{gathered}
c_{1}\left(-\lambda^{2} \sin \lambda L+\lambda\left(m+\gamma i \lambda(i \lambda+\eta)^{\alpha-1}\right) \cos \lambda L\right) \\
=i \lambda f_{1}(L)-m f_{4}-\zeta i \lambda \int_{-\infty}^{+\infty} \frac{\mu(\xi) f_{3}(\xi)}{i \lambda+\xi^{2}+\eta} d \xi-\lambda \int_{0}^{L}\left(f_{2}(\sigma)+i \lambda f_{1}(\sigma)\right) \sin \lambda(L-\sigma) d \sigma \\
+\left(m+\gamma i \lambda(i \lambda+\eta)^{\alpha-1}\right) \int_{0}^{L}\left(f_{2}(\sigma)+i \lambda f_{1}(\sigma)\right) \cos \lambda(L-\sigma) d \sigma .
\end{gathered}
$$

Step 3. We set

$$
g(\lambda)=-\lambda \sin \lambda L+\left(m+\gamma i \lambda(i \lambda+\eta)^{\alpha-1}\right) \cos \lambda L .
$$

As $f_{1} \in H_{L}^{1}(0, L)$ and $f_{2} \in L^{1}(0, L)$, we have

$$
\begin{aligned}
& \left|\int_{0}^{L}\left(f_{2}(\sigma)+i \lambda f_{1}(\sigma)\right) \sin \lambda(L-\sigma) d \sigma\right| \leq c\left(\left\|f_{2}\right\|_{L^{2}(0, L)}+\left\|f_{1}\right\|_{H^{1}(0, L)}\right) . \\
& \left|\int_{0}^{L}\left(f_{2}(\sigma)+i \lambda f_{1}(\sigma)\right) \cos \lambda(L-\sigma) d \sigma\right| \leq c\left(\left\|f_{2}\right\|_{L^{2}(0, L)}+\left\|f_{1}\right\|_{H^{1}(0, L)}\right) .
\end{aligned}
$$

As $g(\lambda) \neq 0$ for all $\lambda$ (if $\eta=0$ then for all $\lambda \neq 0$ ), then $c_{1}$ is uniqueley determined by (5.7). Hence the operator $i \lambda-\mathcal{A}$ is surjective for all $\lambda$ (if $\eta=0$ then for all $\lambda \neq 0$ ). Moreover, taking account of Lemma 4.5, the operator $i \lambda-\mathcal{A}$ is injective for all $\lambda$. Then $i \mathbb{R} \subset \rho(\mathcal{A})$ (if $\eta=0$ then $i \mathbb{R}^{*} \subset \rho(\mathcal{A})$ ).

Moreover, we can easily prove that

$$
|g(\lambda)| \geq c|\lambda|^{\alpha} \text { for } \lambda \text { large. }
$$

Hence

$$
\left|c_{1}\right| \leq c|\lambda|^{-\alpha} \text { for } \lambda \text { large. }
$$


Then, we deduce that

$$
\begin{gathered}
\left\|y_{x}\right\|_{L^{2}(0, L)} \leq c|\lambda|^{1-\alpha} \text { for } \lambda \text { large. } \\
\|v\|_{L^{2}(0, L)} \leq c|\lambda|^{1-\alpha} \text { for } \lambda \text { large. } \\
\quad|z| \leq c|\lambda|^{-\alpha} \text { for } \lambda \text { large. }
\end{gathered}
$$

Moreover from (3.4), we have

$$
\|\phi\|_{L^{2}(-\infty, \infty)}^{2} \leq \int_{-\infty}^{+\infty}\left(\xi^{2}+\eta\right)|\phi(\xi)|^{2} d \xi \leq c\|U\|_{\mathcal{H}}\|F\|_{\mathcal{H}} .
$$

Thus, we conclude that

$$
\left\|(i \lambda I-\mathcal{A})^{-1}\right\|_{\mathcal{H}} \leq c|\lambda|^{1-\alpha} \text { as }|\lambda| \rightarrow \infty .
$$

The conclusion then follows by applying the Theorem 4.2. Besides, we prove that the decay rate is optimal. Indeed, the decay rate is consistent with the asymptotic expansion of eigenvalues which show a behavior of the real part like $k^{-(1-\alpha)}$.

Remark 5.2. The method developed in this paper is direct and very flexible; it can be applied to various dissipative problems. In particular, we will consider in the future more general acoustic wave motions and also multidimensional cases under some geometric control conditions.

Acknowledgement. This research work is supported by the General Direction of Scientific Research and Technological Development (DGRSDT), Algeria.

\section{References}

[1] Achouri, Z., Amroun, N., Benaissa, A., The Euler-Bernoulli beam equation with boundary dissipation of fractional derivative type, Math. Methods Appl. Sci., 40(2017) no. 11, 3887-3854.

[2] Arendt, W., Batty, C.J.K., Tauberian theorems and stability of one-parameter semigroups, Trans. Am. Math. Soc., 306(1988) no. 2, 837-852.

[3] Beale, J.T., Spectral properties of an acoustic boundary condition, Indiana Univ. Math. J., 25(1976), no. 9, 895-917.

[4] Beale, J.T., Acoustic scattering from locally reacting surfaces, Indiana Univ. Math. J., 26(1977), no. 2, 199-222.

[5] Beale, J.T., Rosencrans, S.I., Acoustic boundary conditions, Bull. Amer. Math. Soc., 80(1974), 1276-1278.

[6] Borichev, A., Tomilov, Y., Optimal polynomial decay of functions and operator semigroups, Math. Ann., 347(2010), no. 2, 455-478.

[7] Boukhatem, Y., Benabderrahmane, B., Existence and decay of solutions for a viscoelastic wave equation with acoustic boundary conditions, Nonlinear Anal., 97(2014), 191-209.

[8] Brézis, H., Operateurs Maximaux Monotones et Semi-Groupes de Contractions dans les Espaces de Hilbert, Notas de Matemàtica (50), Universidade Federal do Rio de Janeiro and University of Rochester, North-Holland, Amsterdam, 1973.

[9] Choi, U.J., Maccamy, R.C., Fractional order Volterra equations with applications to elasticity, J. Math. Anal. Appl., 139(1989), no. 2, 448-464. 
[10] Huang, F., Characteristic conditions for exponential stability of linear dynamical systems in Hilbert spaces, Ann. Differential Equations, 1(1985), no. 1, 43-56.

[11] Jiao, Z., Xiao, T.J., Acoustic wave motions stabilized by boundary memory damping, Appl. Math. Lett., 57(2016), 82-89.

[12] Jiao, Z., Xu, Y., Acoustic wave motions stabilized by boundary memory damping II. Polynomial stability, Appl. Math. Lett., 85(2018), 35-40.

[13] Lyubich, Y.I., Phóng, V.Q., Asymptotic stability of linear differential equations in Banach spaces, Studia Math., 88(1988), no. 1, 37-42.

[14] Mbodje, B., Wave energy decay under fractional derivative controls, IIMA J. Math. Control Inform., 23(2006), no. 2, 237-257.

[15] Morse, P.M., Ingard, K.U., Theoretical Acoustics, New York, McGraw-Hill, 1968.

[16] Park, J.Y., Park, S.H., Decay rate estimates for wave equations of memory type with acoustic boundary conditions, Nonlinear Anal., 74(2011), no. 3, 993-998.

[17] Prüss, J., On the spectrum of $C_{0}$-semigroups, Trans. Amer. Math. Soc., 284(1984), no. $2,847-857$.

Khalida Benomar

Djillali Liabes University,

Laboratory of Analysis and Control of PDEs,

P. O. Box 89,

22000 Sidi Bel Abbes, Algeria

e-mail: benomarkhalida@yahoo.com

Abbes Benaissa

Djillali Liabes University,

Laboratory of Analysis and Control of PDEs,

P. O. Box 89,

22000 Sidi Bel Abbes, Algeria

e-mail: benaissa_abbes@yahoo.com 